\title{
ÍNDICE DE AGILIDADE EM PMOS: UM ESTUDO PRÁTICO NO ESTADO DE PERNAMBUCO.
}

\author{
AGILITY INDEX IN PMOS: A PRACTICAL STUDY \\ IN THE STATE OF PERNAMBUCO.
}

Mateus Câmara Pereira ${ }^{1}$

Felipe Santana Furtado Soares ${ }^{2}$

1

Bacharel em Administração de Empresas pela Faculdade de Olinda (FOCCA), Especialista em Gestão de Projetos pela Fundação Getúlio Vargas (FGV-RJ), Especialista em Gestão de Ágil de Projetos pela CESAR School e Mestrando em Ciências da Computação pelo Centro de Informática da Universidade Federal de Pernambuco - CIn UFPE. 2

Bacharel em Engenharia Mecânica pela Universidade Federal de Pernambuco, Mestre e Doutor em Ciência da Computação e Engenharia de Software pelo Centro de Informática da Universidade Federal de Pernamnuco, Diretor Executivo da CESAR School.
RESUMO: Este artigo apresenta o modelo de um índice elaborado para verificar o nível de agilidade dentro das operações de Escritórios de Projetos, Programas e Portfólios (PMO). Para o desenvolvimento desse modelo, foram realizados estudos considerando o contexto histórico, conceitual e tipológico dos PMOs ao longo do tempo. Como também, as principais metodologias e práticas ágeis disseminadas no Mercado global de projetos, servindo assim, como base para estabelecer os critérios, fórmulas e estrutura do modelo. Este artigo, também descreve a experiência dos autores na elaboração desse modelo do índice e a sua aplicação através de uma pesquisa de campo realizada com um grupo composto por 33 PMOs. Estes, pertencentes a empresas de diversos portes e segmentos de negócio, instaladas no Estado de Pernambuco. Demonstrando assim, sua viabilidade e aplicabilidade no Mercado de gerenciamento de projetos. Oportunidades de melhorias foram identificadas, assim como possíveis trabalhos futuros.

Palavras chave: Agilidade; Indicadores; PMO.

ABSTRACT: This paper presents an index model designed to verify the level of agility within Project, Program and Portfolio Office (PMO) operations. To develop this model, studies were conducted considering the historical, conceptual and typological context of PMOs over time. As well as the main methodologies and agile practices disseminated in the global project market, thus serving as a basis for establishing the criteria, formulas and structure of the model. This article also describes the authors' experience in developing this index model and its application through field research conducted with a group of 33 PMOs. These belong to companies of various sizes and business segments, located in the state of Pernambuco. Thus demonstrating its viability and applicability in the project management market. Opportunities for improvement were identified, as well as possible future work.

KEYWORDS: Agility; Indicators; PMO. 


\section{INTRODUÇÃO}

Em meados da década de 90, os primeiros Escritórios de Projetos, PMOs (Project Management Office), começaram a surgir em organizações de todo o mundo (PINTO, 2014). Principalmente, em organizações dos segmentos de tecnologia da informação, engenharia, energia, telecomunicações e serviços. Segmentos de negócios bastantes competitivos dos quais necessitavam de metodologias e processos de gerenciamento focados em projetos, para garantirem o constante incremento de inovações e o respectivo sucesso de seus empreendimentos (BARCAUI, 2012).

Em paralelo a este movimento, em meados dos anos 2000, profissionais da área questionavam a forma tradicional que suas organizações realizavam seus projetos: com um nível exagerado de formalizações, documentações e regulamentações, influenciadas pelo tradicional e burocrático modelo em cascata (BERNADO, 2014). Neste cenário, um grupo de entusiastas e líderes da comunidade do Extreme Programming $(\mathrm{XP})$, se reuniu nos EUA para debater a relação do XP com os "métodos leves" (Lightwirght Methods), avessos aos métodos tradicionais (denominados de métodos pesados), até então empregados nos projetos de desenvolvimento de software daquela época. Desse encontro, surgiu o Manifesto Ágil (BECK, et al, 2001), documento que possui a declaração das crenças, valores e princípios deste grupo, para o desenvolvimento de projetos de software de forma mais fluída e menos burocrática. Ao longo dos anos, estes princípios e crenças ágeis foram incorporadas a novos métodos e frameworks, sendo utilizados por profissionais em diversas organizações, dos mais variados segmentos de negócios, para materialização de seus empreendimentos.

$\mathrm{Na}$ medida que essas práticas foram adotadas no mercado, os estudos elaborados até então, são concentrados apenas a nível projeto, omitindo a aderência e resultados da agilidade no nível dos PMOs. Diante disso, este trabalho se propôs a identificar o nível de agilidade no grupo de PMOs que pertencem a empresas localizadas no Estado de Pernambuco. Para isso, foram analisadas definições, tipologias e contextos de $\mathrm{PMO}$, assim como os métodos e práticas ágeis mais utilizadas no mundo. Em seguida, foi descrita a estruturação do indicador, sua composição, seus critérios, cálculos matemáticos e a lógica do questionário aplicado na pesquisa. Por fim, são apresentadas as algumas análises, resultados, dificuldades, oportunidades de melhorias e possíveis desdobramentos para o futuro deste índice, que foi nomeado de IPMO-A (Índice Project Management Office - Ágil).

\section{TIPOLOGIAS DO PMO}

Levando em consideração os diversos segmentos de negócios e empresas existentes no mundo, a variabilidade de estruturas organizacionais que podem compor uma empresa e seus inúmeros formatos de trabalho, cujos resultados são empreendimentos realizados 
via projetos, surge o Escritório de Gerenciamento de Projetos - EGP, também denominado de Project Management Office - PMO. Trata-se de uma estrutura departamental, responsável pela padronização dos processos de governança relacionados a projetos, pelo compartilhamento de recursos, metodologias, ferramentas e técnicas, para apoiar o gerenciamento de um ou mais projetos dentro de uma organização para entrega de seus resultados (CRUZ, 2016). Diante desse contexto, para identificar um PMO na estrutura organizacional, torna-se importante determinar o seu formato, modelo operacional e grau de influência nos projetos da organização. Segundo Barcaui et. al (2012), o PMO pode ser classificado nas seguintes tipologias:

- Centro de Excelência: fomenta a cultura e apoia os setores na condução dos seus projetos, geralmente, fica ligado a uma gerência departamental e não possui autoridade na gestão dos projetos.

- Escritório de Apoio: realiza tarefas de planejamento e documentação dos projetos que são conduzidos nos setores da organização. Geralmente, responde a um departamento de apoio, ou à própria diretoria, mas possui pouca autoridade na gestão dos projetos.

- Escritório de Projetos: executa tarefas de planejamento e documentação dos projetos em uma estrutura organizacional própria, com gestores de projetos se reportando diretamente para este departamento, possuindo assim, mais autoridade na gestão dos projetos.

- Escritório de Programas: responsável pela gestão integrada dos benefícios, requisitos e riscos da organização, não realiza atividades relacionadas a gestão de projetos. Por ser um órgão de apoio e consulta da presidência, possui grau elevado de maturidade em gestão e influência na estratégia da organização.

- Escritório Executivo: com responsabilidade integral pelos resultados dos projetos, recursos e com poder de decisão, possui grande autoridade e maturidade na gestão do portfólio de projetos da organização, sendo uma diretoria corporativa.

Foi verificado que Aubry e Hobbs (2008), realizaram um trabalho com cerca de 500 PMOs cadastrados na base de dados do Project Management Institute - PMI, classificando o PMO em três tipologias:

- Tipo I: possui grande poder de decisão, reunindo em sua estrutura os projetos da organização e seus respectivos gerentes.

- Tipo II: apesar de reunir em sua estrutura parte dos projetos e alguns gerentes, possui limitação no poder de decisão dos projetos da organização.

- Tipo III: possui alguns projetos sob sua responsabilidade e supervisionam outros, não detém poder de decisão dentro da 
organização.

Para o PMI (2017), a tipologia do PMO pode variar de acordo com seu grau de controle e influência nos projetos da organização, sendo:

- Suporte: desempenha papel consultivo, através da disponibilização de ativos e processos organizacionais, como: modelos, melhores práticas, lições aprendidas e treinamentos, facilitando o acesso das equipes as informações dos projetos.

- Controle: fornece suporte e exige conformidade em sua metodologia, modelos, artefatos, ferramentas e até mesmo a governança dos projetos.

- Diretivo: assume controle total e direto dos projetos, possuindo assim, alto nível de controle dos projetos e portfólio da organização.

Já CRUZ (2016), tipifica o PMO Executor ou Ágil, no qual gerencia e executa diretamente os projetos da organização, com autonomia em suas decisões. Geralmente, este tipo de PMO é aderente a organizações que executam as práticas ágeis, diante da sua adaptabilidade e formato incremental, em relação às práticas tradicionais na gestão e execução dos projetos.

\section{PRÁTICAS ÁGEIS}

Com a mudança de mindset em todos os mercados, mais organizações estão aplicando as metodologias ágeis em seus projetos. Por possibilitar a melhoria contínua de seus processos, entregas com mais valor para seus clientes, mais produtividade a suas equipes e menos burocracia em suas operações. Essas metodologias foram fundamentadas, a partir do Manifesto Ágil (BECK, et al, 2001), onde se busca valorizar:

- Indivíduos e interações mais que processos e ferramentas;

- Software em funcionamento mais que documentação abrangente;

- Colaboração com o cliente mais que negociação de contratos;

- Responder a mudanças mais que seguir um plano.

O termo "Software em funcionamento" pode ser equivalente a "Projeto em funcionamento", estabelecendo assim aderência a qualquer outro segmento de negócio, além do segmento de Tecnologia. Conforme Figura 1, que demonstra os segmentos de negócios dos profissionais que responderam o estudo global publicado no 12th Annual State Of Agile, e atuam no mercado: 


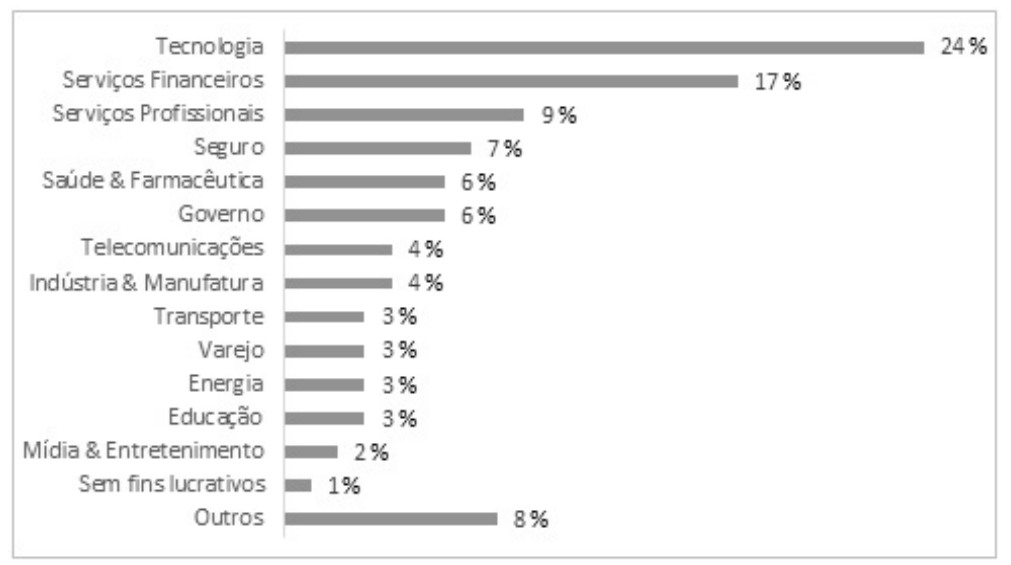

FIGURA 1 - Segmentos de negócios dos profissionais que responderam ao 12th Annual State Of Agile.

FONTE: Adaptada da VERSIONONE (2017).

Dentre os métodos e práticas ágeis mais utilizadas, em 2017, mais da metade dos projetos utilizaram o Scrum (56\%), com bastante aplicação também de metodologias híbridas (14\%), conforme a Figura 2 que demonstra as mais respondidas no 12th Annual State Of Agile:

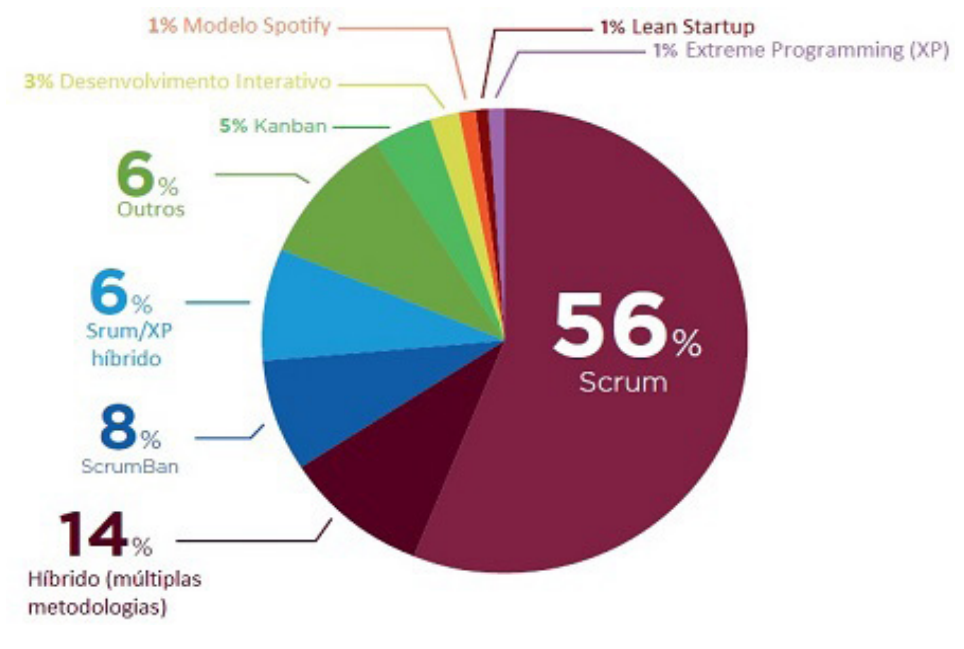

FIGURA 2 - Métodos e práticas ágeis mais utilizadas no mundo.

FONTE: VERSIONONE (2017).

CRUZ (2016, p.43), ainda cita que "- ser ágil, não é ser mais rápido, e, sim, mais eficiente", ficando evidente que essas metodologias e práticas ágeis são disseminadas globalmente. Bem como, utilizadas por diversos profissionais que atuam em variados segmentos de negócios.

\section{FRAMEWORKS ÁGEIS}

Frameworks são conjuntos de conceitos empregados para resolução de um problema, que aplicado na rotina de trabalho, estimula novas metodologias para resolverem questões operacionais comuns a diversas organizações (CRUZ, 2016). Havendo princípios, regras, documenta- 
ções e resultados positivos, podem ser disseminados no mercado para serem utilizadas por qualquer profissional. Os frameworks podem ser utilizados $100 \%$ individualmente, em partes, ou até mesmo misturados (denominamos de híbridos), dentro de um mesmo contexto gerencial. Baseado no entendimento do 12th Annual State Of Agile (VERSIONE, 2017), a seguir são listados os mais relevantes:

- Scrum: foi criado por Ken Schwaber e Jeff Stherland para planejar, gerenciar e desenvolver qualquer produto através de ciclos repetitivos e curtos, de forma interativa e adaptativa aos possíveis desvios durante a execução. Para sua aplicação possui papéis, responsabilidades e cerimônias pré-estabelecidas para a equipe (SOARES, 2015).

- Extreme Programming (XP): elaborado por Kent Beck para a Engenharia de Software, procura dar mais atenção à programação do que ao gerenciamento. Nele, busca-se aprimorar o projeto através da comunicação, simplicidade, feedback, respeito e coragem (SOARES, 2015).

- Lean Startup: estimula o desenvolvimento de ideias prévias de administração e desenvolvimento de produto, incluindo: manufatura enxuta, design thinking como forma de pensar, desenvolvimento de clientes (customer development) e o desenvolvimento ágil (RIES, 2012).

- Modelo Spotify: com times orientados verticalmente (squads e tribos), agrupados por produto (features ou grupos de features) e outros orientados horizontalmente (chapters e guilds), são agrupados por skill ou interesse para propiciar a troca das melhores práticas, experiências, e desafios, com o intuito de obter sinergias internas de conhecimento e produtividade (MELLO, 2018).

-Kanban: busca eliminar estoques e sincronizar a chegada no momento exato dos materiais e seus componentes, ao processo de produção e execução (denominado de just in time), para produzir somente os itens necessários, na quantidade e na hora certa, utilizando cartões e quadro de gestão à vista, fixado nas paredes da organização (SOARES, 2015).

- Scrumban: evolução do Scrum com a inclusão das práticas do Kanban. Esse framework é baseado em um sistema puxado, aonde o refinamento das atividades são realizadas constantemente, de modo que seja mantida as mesmas cerimônias do Scrum de acordo com a necessidade da equipe (PAHUJA, 2018). 


\section{PROCEDIMENTOS METODOLÓGICOS}

Conforme proposta de Barcaui (2003), de forma geral a condução do trabalho obedeceu ao seguinte roteiro básico de pesquisa, como apresentada na Figura 3:

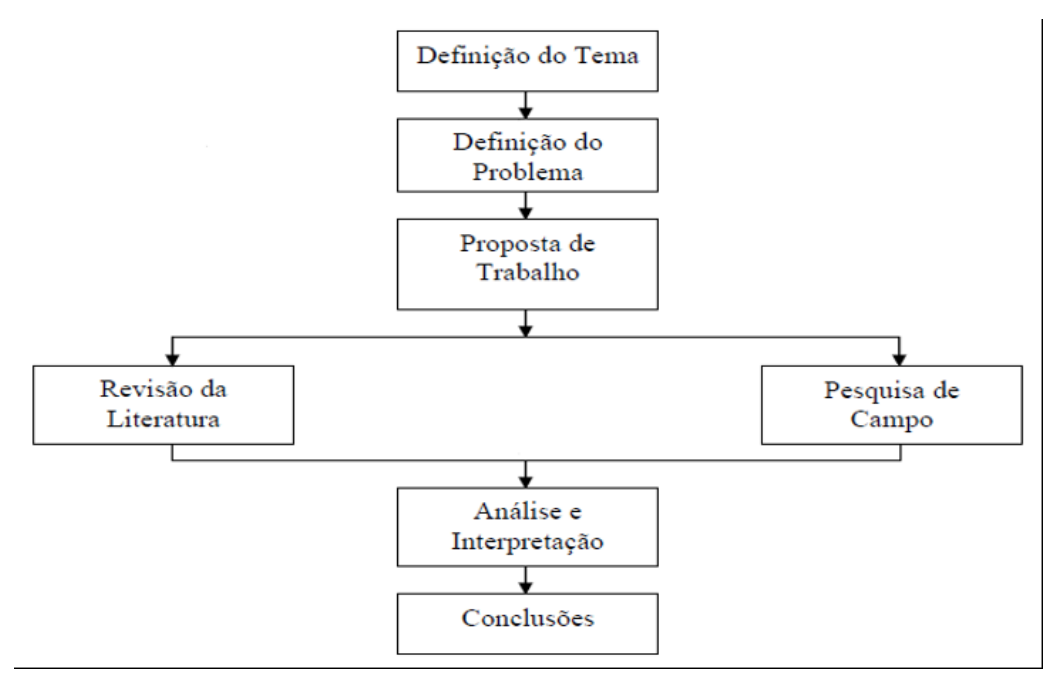

Figura 3 - Roteiro para Pesquisa de Campo.

FONTE: BARCAUI (2003).

Com base na vivência profissional dos autores ao gerenciar projetos em empresas embarcadas no Parque Tecnológico Porto Digital, localizado no Estado de Pernambuco, e experiência na operacionalização de Escritório de Projetos fora deste parque. Foi observado o amplo uso das práticas ágeis a nível projeto, mas não se tinha a visão a nível PMO. Desta forma, surgiram as seguintes perguntas: "Como as práticas ágeis são utilizadas no contexto do PMO?", "Quais os tipos de PMOs utilizam agilidade em suas operações?", e ao delimitar a pesquisa foi estabelecido o seguinte problema: "Qual o nível de agilidade dos PMOs do Estado de Pernambuco?".

A revisão da literatura foi realizada em livros, guias, artigos científicos, dissertações, teses e revistas especializadas relacionadas a temática desta pesquisa. Esta etapa serviu para fundamentar os estudos para elaboração do modelo do índice proposto, confecção do questionário de pesquisa e metodologia no tratamento das informações coletadas para obter o resultado final.

Em seguida, foi realizada uma pesquisa de campo em um grupo real de Escritórios de Projetos no Estado de Pernambuco, pertencentes a empresas que possuem PMOs formalizados em sua estrutura e respondidas por seus respectivos gestores. Na oportunidade, foram identificados os PMOs formalizados e em processo de formalização, sendo identificados ao responderem determinada parte do questionário. $\mathrm{O}$ objeto da pesquisa são os PMOs formalizados, entretanto, também foi possível gerar informações dos futuros PMOs que estão em processo de formalização. 


\section{AMOSTRA DA PESQUISA}

Pernambuco é o sétimo estado mais populoso do Brasil, com cerca de 8.796.032 habitantes e 185 municípios (PERNAMBUCO, 2018). Sua economia, movimenta um Produto Interno Bruto (PIB) estimado em $\mathrm{R} \$ 160$ bilhões, relacionados à agricultura, pecuária, indústria, turismo e serviços. Neste cenário, a gestão de projetos se torna imprescindível para as organizações inseridas nesse contexto, obterem resultados positivos. Em 2003, foi fundado o Pernambuco Brazil Chapter (PMI-PE), primeiro Capítulo da Região Nordeste associado ao PMI Global, com a missão de disseminar e fomentar as boas práticas do gerenciamento de projetos no Estado. Em de 2016, com apoio do PMI-PE, um dos autores deste artigo realizou o Projeto de Mapeamento de PMOs no Estado, resultando na criação de um grupo composto por 33 empresas identificadas na Tabela 1, por segmento de atuação:

\begin{tabular}{|l|c|c|}
\hline \multicolumn{1}{|c|}{ Empresa } & Quantidade & $\mathbf{\%}$ \\
\hline Tecnologia & 10 & 30 \\
\hline Justiça & 6 & 18 \\
\hline Governo & 5 & 15 \\
\hline Comunicação & 2 & 6 \\
\hline Energia & 2 & 6 \\
\hline Indústria & 2 & 6 \\
\hline Saúde & 1 & 3 \\
\hline Saneamento & 1 & 3 \\
\hline Seguros & 1 & 3 \\
\hline Petróleo e Gás & 1 & 3 \\
\hline Ambiental & 1 & 3 \\
\hline Infraestrutura & 1 & 3 \\
\hline \multicolumn{1}{|c|}{ Total } & $\mathbf{3 3}$ & $\mathbf{1 0 0}$ \\
\hline
\end{tabular}

tabela 1 - Distribuição dos PMO mapeados no Estado de Pernambuco. FONTE: Elaborado pelos autores (2019).

Fazem parte deste grupo, gestores e suas respectivas equipes, totalizando atualmente 100 profissionais que se reúnem, periodicamente, em um evento chamado Café com PMO. No qual, a cada encontro, a equipe de um PMO anfitriã recebe in-loco em sua empresa, os demais para compartilhar sua experiência (case), artefatos e ferramentas.

\section{QUESTIONÁRIO DA PESQUISA}

O questionário de pesquisa foi elaborado na plataforma SurveyMonkey ${ }^{\circledR}$ e dividido em 05 (cinco) etapas, conforme resumo a seguir:

- Etapa 01 - Informações Gerais: informa ao participante de que 
se trata a pesquisa, os objetivos esperados e direcionamentos de como respondê-la. Nesta parte, não contém perguntas, sendo exibidas apenas orientações;

- Etapa 02 - Contexto Geral da Organização: coleta informações do participante (nome e cargo) e da organização (nome, segmento, porte e tempo de existência), além de indicar se a organização já formalizou o PMO (há quanto tempo) ou não (em processo de formalização), apresentando uma pergunta chave para direcionar o participante nas próximas etapas do questionário. Nesta etapa, o participante possui a opção de se identificar ou não, assim como a empresa ao qual pertence, garantido assim anonimato;

- Etapa 03 - Contexto do PMO Formalizado: aplicado somente quando o participante informa na Etapa 02 que a organização possui PMO formalizado. Nesta parte, ocorre a coleta de informações do PMO, bem como perguntas referentes aos critérios do índice e seus itens de avaliados. Existem perguntas do tipo múltipla escolha e caixa de seleção, com pontuação ou não (dependendo do item escolhido e resposta efetuada);

- Etapa 04 - Contexto PMO Em Formalização: aplicado somente quando o participante informa que a organização está em processo de formalização do PMO. Nesta parte ocorre a coleta de informações a respeito do futuro do PMO, baseado nos critérios do índice e seus itens de avaliação. Existem perguntas do tipo múltipla escolha e caixa de seleção, e não ocorre pontuação (servindo apenas como estatística);

- Etapa 05 - Finalização: o participante possui a opção de informar seu e-mail (ou não) para envio do resultado da pesquisa, em formato de artigo.

Os possíveis caminhos, etapas e quantidade de perguntas que os participantes do grupo de PMOs responderam, são possíveis visualizar na Figura 4:

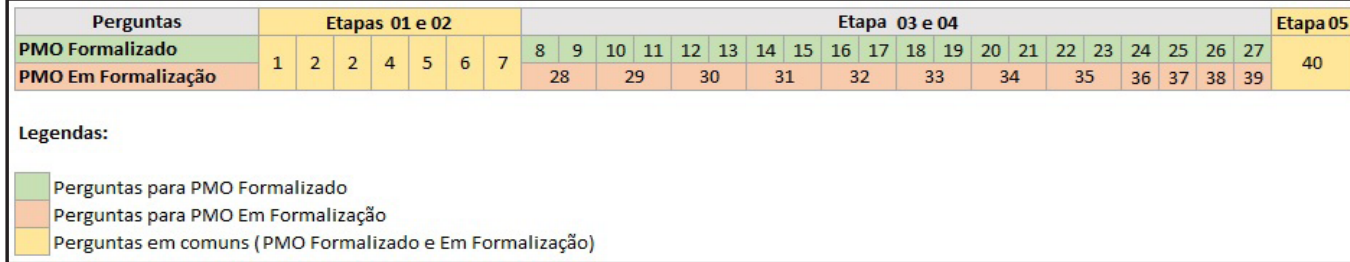

FIGURA 4 - Possíveis caminhos do participante na resposta do questionário.

FONTE: Elaborado pelos autores (2019). 


\section{DELIMITAÇões}

Para estabelecer o índice, foram consideradas algumas delimitações na pesquisa:

- A aplicação da pesquisa foi realizada no território do Estado de Pernambuco, limitando apenas as 33 empresas que fazem parte do Grupo de PMOs do PMI-PE;

- São considerados participantes aptos para participar do índice, aqueles PMOs formalizados;

- A pesquisa leva em consideração ao uso do ágil no contexto operacional do PMO (pela equipe do PMO) e não dentro da organização como o todo (por exemplo no uso dos projetos da organização);

- Considera-se como ágil a sua aplicabilidade, de parte ou por completo, de seus valores, metodologias, práticas e frameworks no contexto do PMO;

- Foram considerados apenas os 04 valores do Manifesto Ágil, que adaptados, podem ser aderentes aos valores do $\mathrm{PMO}$;

- Foram desconsiderados os 12 princípios do Manifesto Ágil, pois são mais aderentes aos projetos do que ao PMO;

- Alguns itens da pesquisa (critérios) são excludentes;

- O formulário foi respondido pelo gestor do PMO;

- Possíveis resultados obtidos pelo PMO através das práticas ágeis, não são objetos de pesquisa desta versão do índice, sem desdobramentos para essas informações.

\section{APLICAÇÃO DO QUESTIONÁRIO}

Após a realização de contatos telefônicos com os respectivos gestores das 33 empresas do grupo de PMOs do PMI-PE, o questionário foi enviado via e-mail junto com o link da pesquisa, conforme a Figura 5:

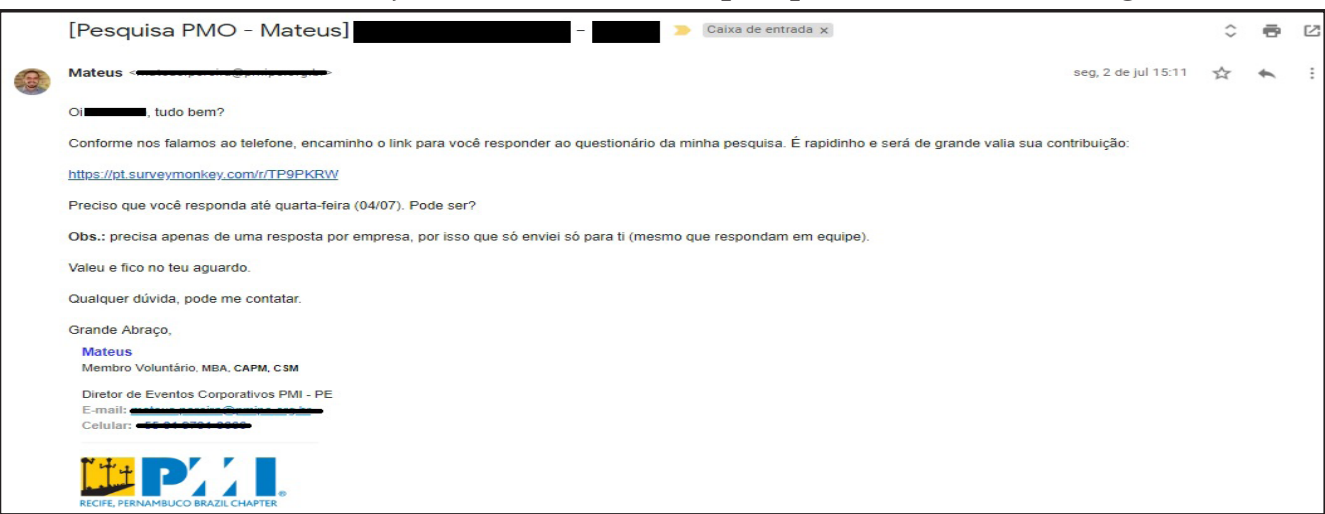

FIGURA 5 - Exemplo do e-mail enviado aos participantes da pesquisa. FONTE: Elaborado pelos autores (2019). 


\section{CRITÉRIOS E PESOS DO ÍNDICE}

O índice possui seis critérios, com objetivos específicos e 74 itens que podem alcançar a pontuação máxima de 47 pontos, sendo eles:

\section{- Critério 01: Valores do PMO}

- Objetivo: identificar se a filosofia e valores de trabalho da equipe na operação do PMO é aderente a algum valor estabelecido no Manifesto Ágil;

- Itens avaliados: 08 (oito);

- Peso: 01 (um).

- Pontuação: até 04 (quatro) pontos;

- Observação: este critério se refere a parte "filosófica" do índice, que possivelmente norteia a equipe do PMO;

- Critério 02: Tipologia do PMO

- Objetivo: verificar se a tipologia do PMO estabelecida para a organização é aderente ao uso de práticas e frameworks ágeis. Neste item, foram consideradas as tipologias disseminadas pelo PMI (2017) e CRUZ (2019), podendo o PMO do tipo de suporte, controle, diretivo e executor (ágil).

- Itens avaliados: 05 (cinco);

- Peso: 02 (dois);

- Pontuação: até 02 (dois) pontos;

- Observação: o tipo de PMO pode facilitar a aderência e uso das práticas ágeis.

\section{- Critério 03: Ferramentas}

- Objetivo: identificar se o PMO utiliza alguma ferramenta (ou artefato) ágil em sua operação.

- Itens avaliados: 11 (onze);

- Peso: 01 (um) e 02 (dois);

- Pontuação: até 11 (onze) pontos;

- Observação: o peso 02 (dois) são para os itens aderentes ao ágil e o peso 01 (um) são para os itens aderentes aos cenários híbrido e tradicional.

- Critério 04: Software

- Objetivo: identificar os softwares utilizados pela equipe do PMO para apoiar suas atividades, gestão do conhecimento e informações do PMO.

- Itens avaliados: 18 (dezoito);

- Peso: 00 (zero);

- Pontuação: 00 (zero); 
- Observação: não existe pontuação nesse critério, pois software não garante adesão a prática ou metodologia ágil na operação do PMO. Este critério serve para coletar outras estatísticas e realizar benchmarking com os participantes para a pesquisa.

\section{- Critério 05: Práticas}

- Objetivo: identificar as principais práticas em gerenciamento de projetos disponíveis no mercado, que possivelmente, o PMO utiliza em sua operação.

- Itens avaliados: 23 (vinte e três);

- Peso: 01 (um) e 02 (dois);

- Pontuação: até 21 (vinte e um) pontos;

- Observação: o peso 02 (dois) são para os itens aderentes ao ágil e o peso 01 (um) são para os itens aderentes aos cenários híbrido e tradicional.

\section{- Critério 06: Framework}

- Objetivo: verificar se o PMO está utilizando um ou mais framework em sua operação.

- Itens avaliados: 09 (nove);

- Peso: 03 (três);

- Pontuação: até 09 (nove) pontos;

- Observação: Peso 03 (três), porque a adoção de um framework ágil é a aceitação e realização das práticas na operação do PMO. Só pontua nesse item, o participante que marcar os itens ágeis.

Em relação aos pesos, foram estabelecidos três possíveis cenários para cada critério, conforme detalhamento a seguir:

- Peso 01, quando o item é aderente somente as metodologias e práticas tradicionais;

- Peso 02, quando o item é híbrido, sendo aderente às metodologias e práticas, tanto ágeis como tradicionais;

- Peso 03, quando o item é aderente somente às metodologias e práticas ágeis.

A composição do índice pode resumida e visualizada de forma completa, conforme a Tabela 2: 


\begin{tabular}{|l|c|c|c|c|c|c|c|}
\hline Composição & Valores & Tipologia & Ferramentas & Software & Práticas & Framework & Total \\
\hline Itens & 08 & 05 & 11 & 18 & 23 & 09 & 74 \\
\hline Peso & 01 & 02 & 01 e 02 & 00 & 01 e 02 & 03 & - \\
\hline $\begin{array}{l}\text { Pontuação } \\
\text { Máxima }\end{array}$ & Até 04 & Até 02 & Até 11 & 00 & Até 21 & 09 & 47 \\
\hline
\end{tabular}

TABELA 2 - Resumo da composição do índice IPMO-A.

FONTE: Elaborado pelos autores (2019).

\section{FÓRMULAS DO ÍNDICE}

De posse dos dados recebidos dos questionários, oriundos dos participantes aptos a mensuração do índice, foram realizados os cálculos em três etapas até chegar ao resultado final, obtendo o índice de agilidade do grupo de PMOs:

- Passo 01 - Identificando o nível do participante: foi realizada a soma de todos os critérios que possuem pesos estabelecidos por participante. É importante citar que o Critério 04, referente a possível utilização de softwares ágeis, não possui peso atribuído. Portanto, não é contemplado no cálculo. A fórmula é a seguinte:

$$
\Sigma \text { critério } 01+\Sigma \text { critério } 02+\Sigma \text { critério } 03+\Sigma \text { critério } 05+\Sigma
$$

- Passo 02 - Identificando o percentual (\%) do participante: foi realizada a divisão entre a soma de todos os critérios com a pontuação máxima possível de obter no índice, conforme cálculo a seguir:

\section{Resultado da $\Sigma$ de todos critérios $\quad=\%$ participante \\ Pontuação máxima possível do questionário}

- Passo 03 - Identificando o percentual (\%) de todo o grupo de participantes: foi realizada a divisão entre a soma de todos os critérios de todos os participantes, com a pontuação máxima de todos os questionários, conforme cálculo a seguir:

$\Sigma$ dos pontos de todos os critérios de todos participantes

$=\%$ do grupo (quantidade de participantes $\mathrm{x}$ pontuação máxima do questionário) 


\section{NÍVEIS DE AGILIDADE}

Soares (2015), verificou que a área de processos de uma organização baseada no modelo CMMI (Capability Matury Model Integration), pode atingir até quatro níveis de agilidade:

- 1 - Inicial: ausência de método ágil para gerenciamento dos projetos ou utilização ad-hoc apenas em poucas práticas isoladas, independente do contexto em que o projeto está inserido, por exemplo stand-up meetings, task-boards, dentre outras.

- 2 - Gerenciado: existe uma gestão ágil de projetos, mas seus processos são caracterizados por projeto. Existem processos ágeis de planejamento e acompanhamento de projeto, mas a visão da organização é por projeto, ou seja, não existe a gestão de programa ou portfólio de projetos.

- 3 - Padronizado: os processos ágeis de gestão de projetos são bem definidos e caracterizados para a organização. Existe um processo padrão de gestão com critérios bem definidos para instanciá-los a cada contexto de um novo projeto.

- 4 - Em otimização: os processos organizacionais de gestão de projetos são, frequentemente, otimizados a partir dos princípios do Lean Software Development, com foco na melhoria contínua.

Com base na sugestão de Soares (2015), e considerando os critérios, lógicas estabelecidas pelos autores para o índice no contexto do PMO em relação a gestão de portfólio de projetos. Os níveis de agilidade foram adaptados para servirem de referência no enquadramento do resultado obtido em percentual (\%), conforme descrições de cada Nível do IPMO-A, na Tabela 3:

\begin{tabular}{|c|l|c|}
\hline Níveis & \multicolumn{1}{|c|}{ Descrição } & IPMO-A \\
\hline 1 - Inicial & $\begin{array}{l}\text { Ausência de valores ágeis na condução do } \\
\text { PMO e poucas práticas ágeis são utilizadas } \\
\text { pela equipe na operação do PMO. }\end{array}$ & Até $15 \%$ \\
\hline 2 - Gerenciado & $\begin{array}{l}\text { Existem alguns valores ágeis na condução } \\
\text { do PMO e pouco uso de ferramentas ágeis } \\
\text { na operação do PMO. A tipologia do PMO } \\
\text { limita o avanço de novas práticas ágeis na } \\
\text { gestão do portfólio da organização. }\end{array}$ & $\begin{array}{c}\text { De } 16 \% \text { a } \\
30 \%\end{array}$ \\
\hline 3 - Padronizado & $\begin{array}{l}\text { O PMO possui os valores ágeis e a equipe } \\
\text { realiza algumas práticas ágeis. A operação } \\
\text { da estrutura é favorecida pela tipologia do } \\
\text { PMO estabelecido para a organização. }\end{array}$ & De 31\% a \\
$75 \%$ \\
\hline $\begin{array}{l}4 \text { - Em } \\
\text { otimização }\end{array}$ & $\begin{array}{l}\text { O PMO segue framezorks ágeis, sua equipe } \\
\text { utiliza bastante as práticas e diversas } \\
\text { ferramentas ágeis na gestão do portfólio dos } \\
\text { projetos da organização. }\end{array}$ & $\begin{array}{c}\text { De } 76 \% \text { a } \\
100 \%\end{array}$ \\
\hline
\end{tabular}

TABELA 3 - Referência dos resultados percentuais (\%) para enquadramento do PMO 
no IPMO-A.

FONTE: Elaborado pelos autores (2019).

\section{RESULTADOS PESQUISA}

Após envio dos 33 questionários para os gestores do grupo de PMOs do PMI-PE, foram respondidos 23 questionários, obtendo assim um bom percentual de participação (70\%), visto que são gestores, possuem pouco tempo em sua rotina para responderem pesquisa e representam empresas importantes do mercado estadual e nacional. Vale salientar, que os participantes que não enviaram suas respostas foram apenas 10, sendo que 2, preencheram o formulário incompleto e 8 não deram retorno algum.

Outras informações gerais das empresas participantes foram coletadas, conforme itens a seguir:

$\checkmark 65 \%$ dos participantes são profissionais que possuem a função de Gerente;

$\checkmark \quad 74 \%$ dos PMOs, pertencem a empresas consideradas de grande porte;

$\checkmark 74 \%$ das empresas, estão há mais de 20 anos em funcionamento no mercado;

$\checkmark \quad 39 \%$ dos PMO, pertencem a empresas do Governo, já que são através de projetos que estes órgãos viabilizam e empregam os recursos para suas ações;

$\checkmark \quad 30 \%$ dos PMOs, pertencem a empresas de Tecnologia, já que o Porto Digital (Parque Tecnológico) foi criado no Estado de Pernambuco e movimenta seus negócios através da realização de projetos.

$\checkmark \quad 82 \%$ dos PMOs, estão formalizados nesse grupo de empresas (objeto da pesquisa) e $28 \%$ em processo de formalização (fora do resultado da pesquisa);

\section{RESULTADOS GRUPO DE PMOS}

Restringindo os resultados para as empresas que possuem PMOs formalizados, objeto dessa pesquisa, foi verificado que $84 \%$ destes PMOs possuem mais de 03 anos de existência e 63\% pertencem a empresas dos segmentos de Governo e Tecnologia, reforçando o cenário descrito nos resultados gerais, nos quais esses segmentos de negócios necessitam de projetos para viabilizar suas operações.

Realizando os Passo 01 - Identificando o nível do participante e Passo 02 - Identificando o percentual (\%) do participante para identificar os índices de cada participante, chegou-se aos resultados individuais, conforme Tabela 4: 


\begin{tabular}{|c|c|c|c|c|}
\hline № & Empresa & $\begin{array}{c}\text { Tipologia do } \\
\text { PMO }\end{array}$ & IPMO-A & Nível \\
\hline 1 & Infraestrutura & De Controle & $9 \%$ & \multirow{4}{*}{1 - Inicial } \\
\hline 2 & Concessionária de Energia & De Suporte & $15 \%$ & \\
\hline 3 & $\begin{array}{l}\text { Desenvolvimento de } \\
\text { Software }\end{array}$ & Diretivo & $15 \%$ & \\
\hline 4 & Prefeitura & De Controle & $15 \%$ & \\
\hline 5 & Indústria de montagem & De Controle & $17 \%$ & \multirow{10}{*}{$\begin{array}{l}2 \text { - } \\
\text { Gerenciado }\end{array}$} \\
\hline 6 & Hospital & De Suporte & $19 \%$ & \\
\hline 7 & Secretaria de Governo & Executor & $19 \%$ & \\
\hline 8 & Justiça Estadual & De Controle & $23 \%$ & \\
\hline 9 & Ambiental & De Suporte & $23 \%$ & \\
\hline 10 & $\begin{array}{l}\text { Desenvolvimento de } \\
\text { Software }\end{array}$ & Diretivo & $23 \%$ & \\
\hline 11 & Indústria automotiva & De Suporte & $28 \%$ & \\
\hline 12 & $\begin{array}{l}\text { Desenvolvimento de } \\
\text { Software }\end{array}$ & De Controle & $28 \%$ & \\
\hline 13 & Secretaria de Governo & De Controle & $28 \%$ & \\
\hline 14 & Justiça Estadual & Executor & $30 \%$ & \\
\hline 15 & Justiça Estadual & De Suporte & $36 \%$ & \multirow{5}{*}{$\begin{array}{c}3 \text { - } \\
\text { Padronizado }\end{array}$} \\
\hline 16 & Seguros & Executor & $45 \%$ & \\
\hline 17 & $\begin{array}{l}\text { Desenvolvimento de } \\
\text { Software }\end{array}$ & Executor & $45 \%$ & \\
\hline 18 & Justiça Estadual & De Controle & $49 \%$ & \\
\hline 19 & Tecnologia para Energia & Executor & $53 \%$ & \\
\hline
\end{tabular}

TABela 4 - Níveis de Agilidade dos participantes aptos que responderam a pesquisa. FONTE: Elaborado pelos autores (2019).

Em seguida foi aplicado o Passo 03 - Identificando o percentual (\%) de todo o grupo de participantes, conforme cálculos a seguir:

$\Sigma$ dos pontos de todos os critérios de todos participantes $=\%$ do grupo (quantidade de participantes x pontuação máxima do questionário)

$$
244 \text { pontos } \quad=28 \%
$$

(19 participantes $x 47$ pontos)

O grupo de PMOs do PMI-PE, representantes do Estado de Pernambuco, obtiveram o IPMO-A de $28 \%$, sendo o Estado enquadrado no Nível 2 - Gerenciado. Quando é realizada a análise por Nível de Agilidade, percebe-se que 53\% dos PMOs obtiveram no Nível 3 Padronizado, sendo a maioria quantitativa e reforçando o resultado anterior, conforme Tabela 5: 


\begin{tabular}{|l|c|c|}
\hline \multicolumn{1}{|c|}{ Níveis de Agilidade } & PMOs & $\mathbf{\%}$ \\
\hline 1 - Inicial & 4 & $21 \%$ \\
\hline 2 - Gerenciado & 10 & $53 \%$ \\
\hline 3 - Padronizado & 5 & $26 \%$ \\
\hline 4 - Em otimização & 0 & $0 \%$ \\
\hline \multicolumn{1}{|c|}{ Total Geral } & $\mathbf{1 9}$ & $\mathbf{1 0 0} \%$ \\
\hline
\end{tabular}

tabela 5 - Níveis de Agilidade dos PMOs de Pernambuco vs Quantidade vs Percentual do grupo.

FONTE: Elaborado pelos autores (2019).

Nota-se que nenhum PMO obteve o nível mais avançado do IPMO-A (Em otimização). E o restante do grupo, ficou dividido nos níveis Inicial e Padronizado, o que demonstra a carência do uso das práticas e metodologias ágeis, bem como a oportunidade de realizar trabalhos para disseminá-las nessas operações. Desta forma, foi possível identificar os Níveis de Agilidades nos PMOs do Estado de Pernambuco.

Ainda foi verificado que em relação a tipologia do PMO, o perfil Executor é mais propenso a utilizar as práticas ágeis, pois obtiveram o Nível 3 - Padronizado com o valor de 38\%, conforme informações na Tabela 6:

\begin{tabular}{|c|c|c|c|}
\hline Tipologias & PMOs & IPMO-A & Nível \\
\hline De Controle & 7 & $24 \%$ & Gerenciado \\
\hline De Suporte & 5 & $24 \%$ & Gerenciado \\
\hline Diretivo & 2 & $19 \%$ & Gerenciado \\
\hline Executor & 5 & $38 \%$ & Padronizado \\
\hline Total & $\mathbf{1 9}$ & - & - \\
\hline
\end{tabular}

TABELA 6 - Índice obtido por tipologia dos PMOs Pernambucanos. FONTE: Elaborado pelos autores (2019).

\section{OPORTUNIDADE DE MELHORIA}

Diante da falta de estatísticas ou informações em agilidade relacionada ao contexto dos PMOs, foi necessário realizar um apurado geral do universo ágil e tradicional, gerando muitos itens para o índice. O que aumentou também, a quantidade de perguntas do questionário, tornando-o cansativo para o participante (preenchimento) e pesquisadores (análise). Como oportunidade de melhoria, realizar a revisão de todos os itens para verificar as perguntas mais relevantes, diminuir a quantidade de itens para tornar o preenchimento do questionário mais agradável e análise dos resultados mais simples.

A amostra principal da pesquisa foram os PMOs formalizados no Estado de Pernambuco. Entretanto, o questionário foi formatado para possibilitar também obtenção das informações dos PMOs que estão em processo de formalização, o que dificultou a compilação dos dados e 
obtenção dos resultados. Como melhoria, redirecionar para outra pesquisa (outro questionário) quando o participante sinalizar que o PMO está em formalização.

E como qualquer índice, a cada nova rodada de aplicação da pesquisa, os resultados sejam analisados, o balanceamento dos percentuais (critérios e pesos) do enquadramento de cada nível do índice sejam calibrados, para que os níveis de agilidade sejam fiéis a realidade nos PMOs.

\section{CONCLUSÃO}

Foi verificado que em Pernambuco mais da metade dos PMOs formalizados $(63 \%)$, pertencem a empresas do segmento de tecnologia, influenciada pela natureza de negócio e pólo tecnológico existente no estado, e do segmento governamental, por ser uma área em que todas as ações e investimentos são viabilizados via projetos. Também foi comprovado que a tipologia escolhida para o PMO, influência fortemente na adoção das práticas ágeis em sua operação. No qual, 60\% dos PMOs que se enquadraram no nível de agilidade 3 - Padronizado, são PMOs do tipo Executor (ou ágil). Foi verificado que nenhum PMO se enquadrou no Nível 4 - Em otimização. Entretanto, 74\% dos PMOs estão enquadrados no Nível - 2 Gerenciado, e apenas $26 \%$ enquadrado no Nível 3 - Padronizado.

O índice IPMO-A, proposto para verificar o nível de agilidade nos PMOs, foi aplicado no Estado de Pernambuco em um grupo de PMOs reais do PMI-PE e funcionou. Também como consequência, foram obtidos: os níveis de agilidade de cada participante, o nível de todos os PMOs (Nível 2 - Gerenciado), considerando assim como o nível de agilidade do estado. Uma vez que estes PMOs estão localizados dentro do território pernambucano e nenhum outro PMO foi identificado até o momento da realização desta pesquisa. As informações desta pesquisa irão gerar um relatório de reporte anual do nível de agilidade em Pernambuco, tendo como benchmark o modelo da VERSIONONE (2017) e o Relatório Técnico RT- MAC-2012-03 de MELO, C.O et al (2012) para demonstrar a utilização das práticas ágeis nos respectivos PMOs e oportunidades de fomentar e disseminá-las para aplicação nessas operações.

Como trabalhos futuros, junto ao PMI Brasil, pode aplicar esta pesquisa em todos os 15 capítulos brasileiros e gerar índices por estado, chegando ao índice Brasileiro do IPMO-A. E ainda aproveitando a rede Global do PMI, pode-se realizar um trabalho internacional de disseminação do índice nos 295 capítulos localizados em 208 países. Bem como outros institutos, órgãos e comunidades ligadas ao ágil, para tornar o IPMO-A um índice de nível global para ser aferido, anualmente, e estimular a adoção das práticas ágeis pelos PMOs ao redor do mundo. Também, pretende-se estimular a criação de novos índices, baseado neste modelo, para as demais áreas do $\mathrm{PMO}$, como por exemplo: maturidade (IPMO-M), riscos (IPMO-R), financeiro (IPMO-F), entre outros, 
consolidando assim, um conjunto de índices denominados IPMO, como referência para escritórios de projetos em qualquer parte do mundo.

\section{REFERÊNCIAS}

AUBRY, Monique; HOBBS, Brian. An empirically grounded search for a typology of Pro-ject management offices. Project Management Journal, v.39, p. 69-82, 2008;

BARCAUI, A. Perfil dos escritórios de projetos em organizações atuantes no Brasil. Dissertação (Programa de Pós-Graduação em Sistema de Gestão).

Universidade Federal Fluminense: Niterói, 2003.

BARCAUI, A. PMO - Escritório de projetos, programas e portfólio na prática. Rio de Janeiro: Brasport, 2012.

BECK, K. et al. Manifesto for Agile Software Development. 2001. Disponível em: <http://www. agilemanifesto.org>. Acesso em 16/09/2019.

BERNARDO, Kleber. Manifesto ágil, como tudo começou. São Paulo: Cultura Ágil, 2014. Disponível em: <https://www.culturaagil.com.br/manifesto-agilcomo-tudo-comecou/ >. Acesso em: 28/09/2018.

CRUZ, Fábio. PMO Ágil: escritório ágil de gerenciamento de projetos. Rio de Janeiro: Brasport, 2016.

KERNZER, Harold. Project Management - A Systems Approach to Planning, Scheduling, and Cotrolling, 10th edition. New York: Van Nostrand Rihold, 2003.

MELO, C. O. et al. Métodos ágeis no Brasil: estado da prática em times e organizações. Relatório Técnico RT- MAC-2012-03. Departamento de Ciência da Computação. IME-USP. Maio, 2012.

MELLO, Francisco Homem de. Como a Spotify organiza seus times de produto. São Paulo, 2018. Disponível em: $<$ http://qulture.rocks/blog/como-a-spotify-organizaseus-times-de-produto/>. Acesso em 01/10/2018.

PAHUJA, Savita. What is Scrumban?. Disponível em: $<$ https://www.agilealliance.org/what-isscrumban/>. Acesso em: 01/10/2018.

PERNAMBUCO, Governo de. Povo de Pernambuco. Recife, 2018. Disponível em: <http://www.pe.gov.br/ conheca/populacao/>. Acesso em: 30/09/2018.

PINTO, A. PMO Insights. Rio de Janeiro: Brasport, 2014. 
PMI, Project Management Institute. Um Guia do Conhecimento em Gerenciamento de Projetos (Guia PMBOK). $6^{\text {a }}$ ed.

Pensilvânia: Project Management Institute, 2017.

RIES, Eric. A startup enxuta: como os empreendedores atuais utilizam a inovação contínua para criar empresas extremamente bem-sucedidas. São Paulo: Lua de Papel, 2012.

SOARES, Felipe Santana Furtado. Uma Estratégia para Implantação de Gestão Ágil de Projetos em Organizações de Desenvolvimento de Software que Buscam Aderência ao CMMI. Tese de Doutorado (Programa de Pós-Graduação em Ciência da Computação) - Universidade Federal de Pernambuco, Recife, 2015.

VERSIONE ONE. 12th Anual State Of Agile ${ }^{\mathrm{TM}}$ Report, 12 volume, 16 páginas, 2017. 